\title{
The effects of energy drink in combination with alcohol on performance and subjective awareness
}

\author{
Chris Alford • Jennifer Hamilton-Morris • \\ Joris C. Verster
}

Received: 30 August 2011 / Accepted: 22 February 2012 / Published online: 29 March 2012

(C) Springer-Verlag 2012

\begin{abstract}
Rationale This study investigated the coadministration of an energy drink with alcohol to study the effects on subjective intoxication and objective performance.

Objectives This study aims to evaluate the objective and subjective effects of alcohol versus placebo at two alcohol doses, alone and in combination with an energy drink, in a balanced order, placebo-controlled, double-blind design. Methods Two groups of ten healthy volunteers, mean (SD) age of 24 (6.5), participated in the study. One group consumed energy drink containing $80 \mathrm{mg}$ of caffeine and the other consumed a placebo drink, with both receiving two alcohol doses ( 0.046 and $0.087 \%$ breathalyser alcohol concentration). Tests included breath alcohol assessment, objective measures of performance (reaction time, word memory and Stroop task) and subjective visual analogue mood scales.

Results Participants showed significantly impaired reaction time and memory after alcohol compared to the no alcohol condition and had poorer memory after the higher alcohol dose. Stroop performance was improved with the energy drink plus alcohol combination compared to the placebo drink plus alcohol combination. Participants felt significant subjective dose-related impairment after alcohol compared
\end{abstract}

C. Alford $(\triangle) \cdot J$. Hamilton-Morris

Psychology Department, Faculty of Health and Life Sciences, University of the West of England,

Frenchay Campus, Coldharbour Lane,

Bristol BS16 1QY, UK

e-mail: chris.alford@uwe.ac.uk

J. C. Verster

Division of Pharmacology, Utrecht Institute for Pharmaceutical

Sciences, Utrecht University,

Universiteitsweg 99 ,

3584 CG, Utrecht, The Netherlands to no alcohol. Neither breath alcohol concentration nor the subjective measures showed a significant difference between the energy drink and the placebo energy drink when combined with alcohol.

Conclusions Subjective effects reflected awareness of alcohol intoxication and sensitivity to increasing alcohol dose. There were no overall significant group differences for subjective measures between energy drink and placebo groups in the presence of alcohol and no evidence that the energy drink masked the subjective effects of alcohol at either dose.

Keywords Alcohol - Antagonism · Caffeine - Energy drink . Intoxication $\cdot$ Performance $\cdot$ Subjective awareness

\section{Introduction}

Energy drinks are sold in more than 160 countries, and sales are increasing. Energy drinks contain caffeine, taurine and carbohydrates in the form of sugars as principal ingredients (Heckman et al. 2010). They are popular with young people, and students report their use for a variety of reasons including to enhance memory and concentration or to counteract sleepiness (Malinauskas et al. 2007; O'Brien et al. 2008). Whilst a minority of students combine energy drinks with alcohol (Arria et al. 2010; Attila and Cakir 2010; Berger et al. 2010; Malinauskas et al. 2007; O'Brien et al. 2008; Oteri et al. 2007; Rossheim and Thombs 2011), this still represents a significant number and so they are becoming a popular mixer. Caffeine and alcohol are two of the oldest commonly consumed psychoactive compounds, and caffeinated drinks have been mixed with alcohol for many years. 
There are some recent claims that energy drinks may mask the subjective effects of alcohol, and this may either lead people to drink more or that they may be unaware of how intoxicated they are (Arria et al. 2010; Arria and O'Brien 2011; O'Brien et al. 2008), although these views on masking have been challenged (Verster and Alford 2011) and an alternative explanation for enhanced intoxication with novel drink flavours has been offered by Siegel (2011). A brief review of the effects of caffeine and energy drinks alone and in combination with alcohol is provided in order to establish whether or not energy drinks appear different to other caffeinated drinks when mixed with alcohol.

\section{Mechanisms of action}

Folklore tells how caffeine has long been used as a stimulant to help maintain wakefulness, including helping monks with their nightly prayers, and early studies found that caffeine improved performance including the speed of response (Hollingworth 1912; Ritchie 1980; Schilling 1921). The primary action of caffeine as an adenosine inhibitor is well established, thus counteracting the actions of this inhibitory neurotransmitter and increasing the release of other neurotransmitters including noradrenaline and dopamine, producing a moderate stimulant and mood-enhancing effect. Blockade of the $\mathrm{A}_{2 \mathrm{~A}}$ receptor by caffeine inhibits the sleeppromoting effect with adenosine levels increasing across the hours of wakefulness (Davis et al. 2003; Ferré 2008; Huang et al. 2007; Landolt 2008; Sturgess et al. 2010).

In contrast to caffeine, alcohol has long been found to have detrimental effects on both judgement and performance (Cohen et al. 1958; Wallgren and Barry 1970), with Miller (1992) concluding, 'In general, alcohol has an adverse effect on cognitive performance'. Alcohol may have more complex effects than caffeine, with regions of the CNS affected differentially by dose (Rivers 1994) and with multiple actions including depressing the ion channel function controlled by the excitatory neurotransmitter glutamate at the NMDA receptor, inhibiting acetylcholine release and potentiating GABA resulting in impaired cognition, inability to form new memories and sedation (Braun 1996; McKim 1997). Alcohol has a wide spectrum of action within the CNS affecting many neurotransmitter systems including major neurotransmitters adenosine, dopamine, GABA, glutamate and serotonin. Interaction with the mesolimbic dopamine reward pathways may well contribute to the process of addiction and alcohol misuse (Lovinger 2008). The comparatively restricted action spectrum of caffeine, focused on adenosine inhibition, may account for its more limited misuse potential seen in caffeinism, although it is not recognized as a dependence-inducing substance (APA 1994).

\section{Effects on subjective state and performance}

Alcohol impairs a wide range of psychomotor and cognitive tasks, slowing reaction times, impairing memory formation and increasing errors (Cameron et al. 2001; Hindmarch et al. 1991; Mackay et al. 2002; Maylor and Rabbitt 1993; Millar et al. 1995; Moskowitz et al. 1985; Ogden and Moskowitz 2004; Tiplady et al. 2009). The differential effects on regions of the CNS may be linked to lower doses affecting higher-level processing (Jääskeläinen et al. 1995) and genetic differences contribute to overall variation in sensitivity to alcohol (Tagawa et al. 2000). Koelega (1995) concluded that the effects on information processing and divided attention tasks are important because of their relevance to everyday life. Therefore, it is not surprising that alcohol impairs driving and driving-related skills (Holloway 1995; Starmer 1989; Verster et al. 2009). The dose-response curve linking blood alcohol level and relative probability of causing a driving accident has long been established and applied in setting legal limits for alcohol levels and driving (Borkenstein et al. 1964) with limits in Europe, US and UK currently set around $0.05-0.08 \%$ blood alcohol concentration (BAC) levels. Laboratory studies show that driving-related skills are significantly impaired at the $0.05 \%$ BAC level (Ogden and Moskowitz 2004).

Alcohol intoxication has been linked to riskier sexual behaviour (Fromme et al. 1999), although there may problems with the use of retrospective accounts (Halpern-Fischer et al. 1996). Judgement may also be affected with people overestimating their abilities (Flanagan et al. 1983; Tiplady et al. 2004), including being able to deal with a potentially risky situation or acting on immediate short-term consequences rather than longer-term consequences (Farquhar et al. 2002). Specific to driving, alcohol may cause a 'risky shift', resulting in hitting the test bollards and failing the gap acceptance test (Alford and Starmer 1990, personal communication; Betts et al. 1984).

In contrast to alcohol, the moderate stimulant effects of caffeine are associated with improved performance. Doses typically in the 100- to 200-mg dose range result in improved mood (Mumford et al. 1994), shorter reaction time and improved attention and vigilance (Frewer and Lader 1991; Koelega 1998; Nehlig et al. 1992; Robelin and Rogers 1998; Smit and Rogers 2000; Smith 2002; Van der Stelt and Snel 1998) in a dose-dependent way (Smith et al. 2005). However, significant effects are not always seen, which may be due to the limited effect size and possible ceiling effects in that it is harder to enhance performance in healthy young adults. There is also genetic variation in response to caffeine (Nehlig 2010) so that sampling will affect observed response. Caffeine and glucose are both contained in energy drinks, and studies have found improved performance when they are taken together. Both Adan and Serra-Grabulosa 
(2011) and Scholey and Kennedy (2004) found faster speed of response with attentional tasks, as well as improved memory, with caffeine and glucose combined in comparison to the individual constituents, although significant changes in mood were not seen.

Overnight withdrawal from caffeine can itself result in improved performance following morning caffeine: the caffeine reinstatement or withdrawal hypothesis (James 1997; James and Rogers 2005), although improvements in performance are still seen independently of this when additional caffeine is consumed after the usual caffeine intake (Christopher et al. 2005; Smith et al. 2005; Warburton 1995; Watson et al. 2002). Beneficial effects are more clearly seen when participants are sleep-deprived or fatigued (Alford 2009; Davis et al. 2003; Fagan et al. 1998; James 1997; James and Gregg 2004; Ker et al. 2010; Nehlig 2010; Rogers et al. 2005; Smith 2002; Swift and Tiplady 1988), with a recent review (Glade 2010) concluding that caffeine has a beneficial effect increasing energy availability and expenditure whilst decreasing the subjective effort linked to physical activity, enhancing physical, motor and cognitive ability including neuromuscular coordination, speeding and increasing accuracy of reactions, increasing concentration, focus and short-term memory and problem solving, increasing correct decision making, and increasing subjective feelings of alertness and energy (for physical performance, see Astorino and Roberson 2010; Sokmen et al. 2008).

\section{Effects of energy drinks}

Since their introduction into Europe in the late 1980s, there has been interest in the performance-enhancing effects of energy drinks. Popular brands contain around $80 \mathrm{mg}$ of caffeine per $250 \mathrm{ml}$ which places them above colas but on a similar level to coffee, although some minority brands can contain higher doses as can other preparations of coffee (IFIC 1998; Reissig et al. 2009). Other key ingredients include taurine, carbohydrates in the form of sugars and glucoronolactone (a sugar metabolite) and B complex vitamins.

Energy drink consumption has been shown to improve physical performance in several but not all studies (Alford et al. 2001; Hoffman 2010; Ivy et al. 2009; Klepacki 2010; Walsh et al. 2010). In addition, improvements have been shown for both psychomotor and cognitive functioning, as well as driving and driving-related skills (Alford et al. 2001; Gershon et al. 2009; Horne and Reyner 2001; Mets et al. 2010; Reyner and Horne 2002; Seidl et al. 2000; Smit et al. 2006).

\section{Caffeine and alcohol}

Caffeine and alcohol are commonly combined either through consumption of alcohol with caffeinated mixers including colas or energy drinks (Thombs et al. 2010a, b) or through proximal consumption of alcohol and caffeine such as dinner with alcohol followed by coffee. Given that caffeine is a weak stimulant and alcohol is a sedative, a predicted outcome would be that caffeine would antagonize the effects of alcohol, reducing both subjective and objective impairment. However, whilst some studies have shown antagonism (Hasenfratz et al. 1993), some others have found no effect (Nuotto et al. 1982) and yet others have shown that caffeine may even increase the impairing effects of alcohol (Oborne and Rogers 1983). A recent view is that caffeine can antagonise some, but not all, behavioural effects of alcohol (Azcona et al. 1995; Liguori and Robinson 2001; Marczinski and Fillmore 2006), although expectancy may be involved (Fillmore et al. 2002; Fillmore and VogelSprott 1995). Azcona et al. suggested that caffeine antagonism may more readily be seen in tasks where caffeine itself has a more positive effect on performance.

An important safety-related aspect of psychopharmacological investigations is whether or not subjective awareness parallels objective impairment or behavioural toxicity (Hindmarch et al. 1992). Laboratory investigations have shown that caffeine and alcohol in combination do not alter the 'high' or perceived or actual BAC with caffeine levels up to around $400 \mathrm{mg}$ and alcohol $0.012 \%$ BAC (Howland et al. 2010; Liguori and Robinson 2001; Rush et al. 1993).

\section{Energy drinks and alcohol}

Given the increasing popularity of energy drinks and that caffeine is a principal ingredient, it is logical to raise questions regarding their interaction with alcohol on both subjective and objective measures. A recent commentary claimed that individuals who combined energy drinks with alcohol underestimate their true level of impairment and that the drink combination could lead to engaging in risky behaviour, whilst others have claimed that these effects could have an increased accident risk (Arria and O'Brien 2011; Riesselmann et al. 1996).

Currently, there are only a few studies investigating the combined effects of energy drink and alcohol compared to alcohol alone. Ferreira et al. (2004) investigated the effects on physical performance of alcohol $(1.0 \mathrm{~g} / \mathrm{kg})$ and energy drink ( $3.57 \mathrm{ml} / \mathrm{kg}$ equivalent to a standard $250 \mathrm{ml}$ can $)$ alone and in combination. Whilst alcohol impaired maximal effort assessed using a cycle ergometer, no significant differences were found between alcohol alone and energy drink combined with alcohol. A recent study by Marczinski et al. (2011) compared the effects of alcohol alone to alcohol combined with energy drink at $0.06-0.07 \%$ breath alcohol concentration (BrAC). They found no differences in breath alcohol or level of impaired performance. They undertook over 20 individual subjective ratings, including perceived intoxication and ability to drive, which again found no 
differences between alcohol and energy drink in combination with alcohol. However, the authors focused in on two subjective measures that failed to show significant effects with ANOVA but did show reduced mental fatigue and increased stimulation for the energy drink and alcohol combination compared to alcohol, but not against placebo for the latter, using paired comparisons. They interpreted this as potentially dangerous despite the lack of difference in a direct subjective measure of intoxication, as well as ability to drive.

Several authors have recently claimed that energy drink when combined with alcohol produces a reduction in perceived intoxication or 'masking' of subjective awareness (Arria and O'Brien 2011), although this appears to be based on a single study which is, therefore, considered in some detail.

Ferreira et al. (2006) compared the effects of two doses of alcohol (0.04-0.05 and 0.097-0.099\% BrAC) assessed using two participant groups, when given alone and in combination with energy drink. Psychomotor performance was assessed (reaction time and grooved peg board) and found to be impaired with both alcohol alone and the alcohol and energy drink combination. An 18-item modified somatic symptom scale assessed subjective intoxication at 2 time points $(18 \times 2$ scales $)$. Dry mouth, headache, motor impairment and weakness (4), i.e. not all were typical alcohol impairment assessment scales, showed reduced perception for the alcohol energy drink combination compared to alcohol at 1 of the 2 time points $(4 / 18 \times 2)$ for alcohol doses combined. More scales, though including 2 from the above: dizziness, motor impairment, speech, tiredness, vision, walking, weakness and well-being (8), showed impairment with alcohol at more time points $(10 / 18 \times 2)$ that were not reduced for the alcohol and energy drink combination. However, the authors concluded that energy drink reduced the perception of alcohol impairment.

Given the variability of results regarding the perception of impairment, further studies are required to clarify the effects of energy drink when combined with alcohol on the subjective perception of intoxication. The current study examined these effects with the use of both objective performance measures to establish impairment and subjective ratings to assess perceived impairment.

\section{Methods}

\section{Design}

The present study investigated the effects of alcohol alone and in combination with an energy drink in a placebocontrolled, rising dose, double-blind design. There were two groups of participants with random allocation to treatment. One group received energy drink and the other received placebo energy drink in a balanced order for both the alcohol and placebo alcohol treatments on two separate occasions.

\section{Participants}

Ten female and 10 male participants provided 2 mixed gender groups of 10 volunteers, with the mean age of 24.5 years and range of 19-33 years. Average weight was $70.7 \mathrm{~kg}$; for these light to moderate social drinkers, range was 4-30 units, averaging 17 units per week (females 13.4, males 20.6), with daily caffeine of $350 \mathrm{mg}$. Prior to participation, the study was approved by a University of the West of England Ethics Committee and each participant signed an informed consent. All participants reported that they were in good health, not pregnant, taking illicit or social drugs, 'over the counter' or prescribed medication with the exception of the contraceptive pill. None reported adverse reactions to either alcohol, caffeine or energy drinks and all consumed weekly alcohol and had tried an energy drink at least once, but not on a regular basis.

\section{Assessments}

Breath alcohol concentration Alcometer readings were taken by the experimenter who kept their hand over the visual display so that participants were blind to their alcohol concentration readings.

Subjective impairment was measured using the Bond and Lader (1974) 100-mm visual analogue scales (VAS) representing bipolar adjective pairs for the assessment of both alerting and sedating CNS drug effects with clearheadedmuzzy, clumsy-well coordinated, energetic-lethargic, drowsy-alert, and mentally slow-quick witted selected for analysis of alcohol-induced impairment. These scales have previously been used to assess both energy drinks and alcohol (Alford et al. 2001; Tiplady et al. 2004; Warburton et al. 2001).

Critical flicker fusion threshold This was assessed by viewing four red-coloured light-emitting diodes set $1 \mathrm{~cm}$ apart in a square formation on a black background providing foveal fixation when viewed at $1 \mathrm{~m}$. The diodes flickered on/off at a constantly varying rate of $1 \mathrm{~Hz} / \mathrm{s}$ with participants pressing a response button at the point of perceiving either fusion or flicker for three ascending and descending repetitions using the psychophysical method of limits (Hindmarch et al. 1991).

Choice reaction time Psychomotor speed was measured using a free standing box which comprised a touchsensitive home pad around which six equally spaced 
response pads were situated at $150 \mathrm{~mm}$ from the home pad in a $120^{\circ}$ arc with a red light-emitting signal diode positioned distal to each response pad. Participants were required to move their finger from the home pad to the appropriate pad as quickly and accurately as possible in response to illumination of one of the stimulus diodes which were lit in a random sequence over 20 trials (Hindmarch et al. 1991).

Word memory Printed sheets each containing a vertical list of 30 words based on Pavio's word lists balanced for concreteness, imagery and meaningfulness were used to assess memory (Paivio et al. 1968). Participants had $1 \mathrm{~min}$ to view and memorise the list, followed by a further minute to write down as many words as they could from the original list.

Stroop cognitive interference task (Stroop) Participants were required to say out loud the colour of the ink, and not the printed word, whilst reading down each of 4 columns totalling 100 printed colour words. For example, with the word 'RED' printed in green ink, a correct response would be to say 'green', ignoring what the letters spelt (Stroop 1935). The time taken to complete the list and the number of errors was recorded.

\section{Equipment}

Critical flicker fusion (CFF) and choice reaction time (CRT) were tested using dedicated hardware supplied by Comstat Medical based on the Leeds psychomotor tester (Hindmarch et al. 1991). \%BrAC was used to estimate \%BAC using a Lion Alcometer SD-400, Lion Laboratories, Barry, UK.

\section{Treatments}

Alcohol was given as vodka $(37.5 \%$ by volume) with amount adjusted for body weight using the formula adapted from McKim (1997) to achieve an estimated blood alcohol level of approximately $0.1 \%$ BAC divided across two drinks. The energy drink treatment comprised a standard can of Red Bull ${ }^{\circledR}$ Energy Drink $(250 \mathrm{ml})$ containing $80 \mathrm{mg}$ caffeine to which was added peppermint cordial and Robinson's apple and black currant (no added sugar) concentrate to mask the flavour of the energy drink. The placebo comprised $250 \mathrm{ml}$ soda water together with peppermint cordial and Robinson's apple and black currant (no added sugar) concentrate with equal total volumes for alcohol and non-alcohol conditions. Supercook black/blue food colouring was added to help mask the presence of energy drink, with two drinks presented in each treatment condition in glasses after the rim was swabbed with alcohol to help further mask treatments.
Procedure

An opportunity sample of potential participants completed screening questionnaires to assess health status, exclude nondrinkers, excessive drinkers, none or high caffeine users, or experience of an adverse reaction to test compounds, or those who may be pregnant. On test days, health status and the preceding night's sleep was checked. They were asked to abstain from alcohol and caffeine from the preceding night, not drive to the university on test days, and have a light lunch an hour before testing which was undertaken in the psychology laboratories starting between 1 and $2 \mathrm{pm}$. A practice session, weighing, explanation of study procedures and random allocation to treatment group preceded testing on the first test day with test sessions averaging $2.5 \mathrm{~h}$ for the two test days which were separated by a week. Treatments were administered double-blind with the assistance of an additional experimenter who prepared the drinks. Participants were assessed three times on each test day, first at baseline then 45 min after starting the first drink, with $10 \mathrm{~min}$ for drinking, and similarly after the second with about an hour between drinks. The fixed test order, though different word lists, started with subjective VAS assessment, then breathalyser, followed by word list memory, Stroop, CFF, CRT and finally delayed memory recall all administered with standardised instructions. After completing the test sessions, participants were escorted home and later followed up to check they had not experienced any adverse reaction to treatment. Debriefing followed the final test day.

\section{Data handling}

All data were analysed blind. Raw data were collated and transcribed into Excel spreadsheets. The Comstat Medical equipment provided numerical values for ascending and descending CFF thresholds, resulting in an overall mean. Similarly, separate component means were given for CRT including recognition reaction time (RRT) representing elapsed time from stimulus signal onset to initiation of response when the finger is lifted from the home pad. Movement reaction time (MRT) is the time taken for the finger to be moved from the home pad to the target pad and total CRT is the sum of these two elements (RRT + MRT) combined. For word memory, the correct number recalled within the $60 \mathrm{~s}$ immediate or delayed recall period was entered. For the Stroop task, the number of errors and time taken to complete the task were recorded. The VAS scales assessing subjective impairment were scored by measuring the distance along the $100-\mathrm{mm}$ line separating the adjective pairs (e.g. drowsy-alert) for each of the five adjectives. These values were also entered onto the results spreadsheet together with the alcometer readings which were manually recorded at each breath analysis session. 


\section{Results}

Statistical analysis

Data were analysed using SPSS V17 (SPSS 2010). Placebo baselines were compared for the energy drink and placebo energy drink groups with $t$ tests. Neither subjective impairment measures nor performance measures produced significant contrasts, demonstrating the comparability of the two participant groups. To help standardise results between the two groups of participants (energy drink and placebo energy drink), as well as helping to control for variation between test days, baseline difference scores were calculated. These scores were then analysed using a three-factor $2 \times 2 \times 2$ ANOVA (treatment group 2: energy drink and placebo energy drink; alcohol 2: alcohol and placebo alcohol; drinks 2: drink 1 and drink 2). Statistical significance was set at the $5 \%$ level $(P<0.05$ or better) to assess main effects and interactions. The analysis was, therefore, focused on assessing the overall effects of alcohol (alcohol vs placebo alcohol), increasing dose (drink 1 vs drink 2) and energy drink (energy drink vs placebo energy drink). A simple main effects analysis was additionally employed to specifically assess differences between alcohol and energy drink contrasted with alcohol and energy drink placebo. A summary table including means (SEM), together with associated $P$ values and effect sizes, for both subjective and performance measures are included in Table 1.

\section{Alcohol dose}

All participants produced zero BrAC at each baseline and for the placebo alcohol treatment. Mean BrAC after drink 1 was $0.046 \%$ BrAC and after drink 2 was $0.087 \%$ BrAC. The mean (SD) results for the energy drink group were 0.044 $(0.01)$ and $0.081(0.02) \% \mathrm{BrAC}$ and for the placebo energy drink group were $0.047(0.01)$ and $0.094(0.02) \%$ BrAC after drinks 1 and 2, respectively. Differences between treatment groups were nonsignificant ( $t$ test), facilitating comparison between the two groups for alcohol versus placebo and between drinks 1 and 2, though noting highest breath alcohol levels were recorded after drink 2 for the placebo energy drink group.

\section{Performance tests}

\section{Critical flicker fusion}

Whilst no significant main effects or interactions were found, the lowest thresholds were recorded in the alcohol condition for both groups, consistent with a sedative effect.

\section{Choice reaction time}

Alcohol slowed total CRT, although differences failed to achieve significance for the main effects of alcohol and drink; the energy drink produced a trend $(P<0.1)$ for faster responses compared to placebo. RRT showed a significant slowing after alcohol $\left(F_{1,18}=6.5, \mathrm{MSe}=0.00, P=0.02\right)$ and trends for faster responses with the energy drink $(P<0.1)$ and for an interaction between alcohol and drink $(P<0.1)$, reflecting slower responses after the second alcohol drink compared to faster responses after alcohol placebo for both the energy drink and placebo energy drink groups combined. Means are plotted in Fig. 1.

\section{Word memory}

Similar effects were found for both immediate and delayed word memory recall. Alcohol markedly reduced the number of words recalled (immediate: $F_{1,18}=23.8, \mathrm{MSe}=12.3, P=$ 0.001; delayed: $\left.F_{1,18}=23.9, \mathrm{MSe}=19.5, P=0.001\right)$ and in a dose-dependent way (drinks-immediate: $F_{1,18}=28.6$, $\mathrm{MSe}=3.46, P=0.001$; delayed: $F_{1,18}=22.0, \mathrm{MSe}=7.52$, $P=0.001$ ), resulting in an alcohol $\times$ drink interaction reflecting the decrease in words recalled with increased alcohol compared to alcohol placebo (interaction-immediate: $F_{1,18}=15.8, \mathrm{MSe}=6.57, P=0.001$; delayed: $F_{1,18}=18.0$, $\mathrm{MSe}=13.0, P=0.001)$. These highly significant effects for immediate recall are shown in Fig. 1.

\section{Stroop task}

Error rate was not significantly affected by alcohol overall, although the highest error scores were recorded after the second alcohol drink for the placebo energy drink group and with a trend for lower error scores in the energy drink group $(P<0.1)$. Stroop completion time was faster with energy drink $\left(F_{1,18}=11.1, \mathrm{MSe}=0.035, P=0.004\right)$ and unexpectedly faster still for alcohol combined with energy drink, resulting in a main effect for alcohol $\left(F_{1,18}=6.06, \mathrm{MSe}=0.03, P=0.024\right)$, as well as a significant interaction between alcohol and treatment group $\left(F_{1,18}=4.59, \mathrm{MSe}=0.03, P=0.046\right)$. Simple main effects analysis revealed that, in addition to completion time $\left(F_{1,18}=11.2, \mathrm{MSe}=0.05, P=0.004\right)$, Stroop errors were also significantly reduced $\left(F_{1,18}=5.71, \mathrm{MSe}=3.68, P=0.028\right)$ by alcohol combined with energy drink contrasted with the alcohol and energy drink placebo combination.

Effect sizes (Cohen's $d$ ) can be characterised as follows: small $d=0.2$; medium $d=0.5$; large $d=0.8$ (Cohen 1992). Effect sizes of $d \geq 0.2$ were generally associated with statistically significant results, although both CRT overall and RRT produced effect sizes for energy drink contrasted with energy drink placebo of just over $d=0.2$, reflecting faster responses after energy drink that approached significance $(P=0.06)$. 


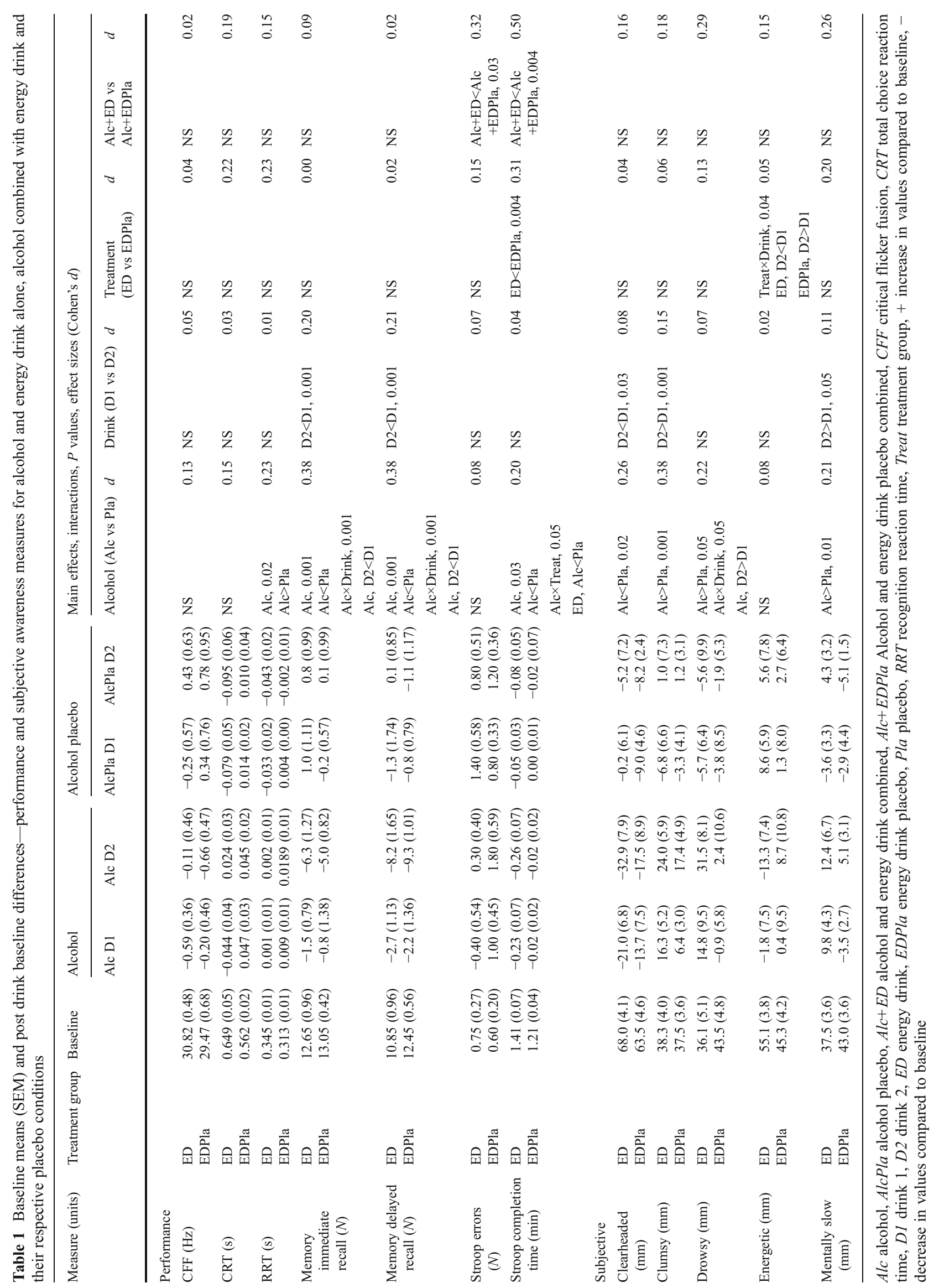




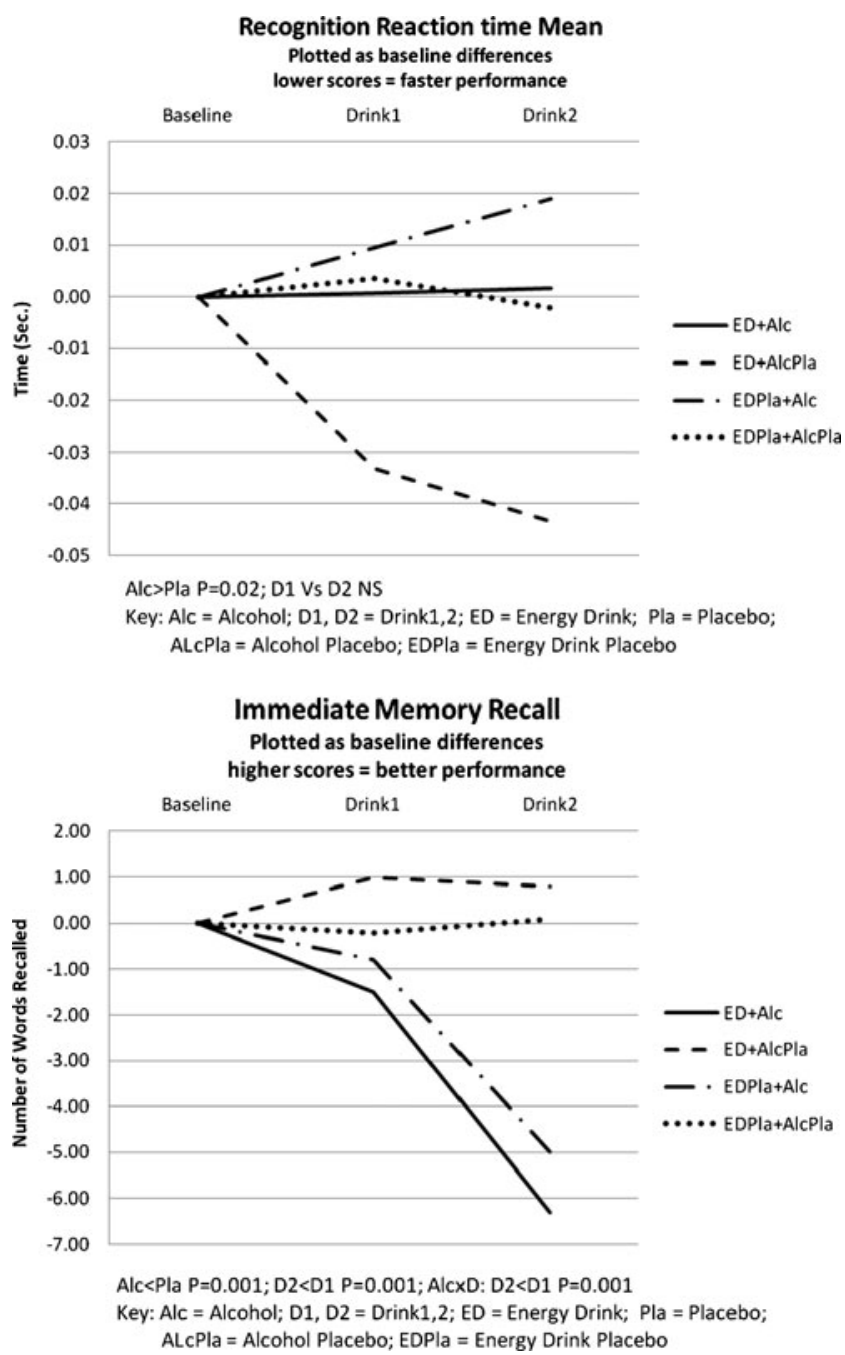

Fig. 1 Performance results - RRT and immediate memory recall, contrasting alcohol and energy drink alone, as well as in combination, and against their respective placebos

In summary, alcohol significantly impaired both reaction time and memory performance. The Stroop task results were more complex. Whilst alcohol was associated with the most errors in the placebo energy drink group and completion time was faster after energy drink, the combination of energy drink and alcohol produced the fastest completion times, as well as reducing errors.

Therefore, either trends or significant impairments were seen with alcohol for five out of seven variables derived from the four performance tests. The most significant effects were seen with memory, including a dose-related increase in impairment with alcohol. The effects of energy drink were relatively weak in overcoming the effects of alcohol so that only one (Stroop) out of four separate tests resulted in a significant improvement with energy drink, reflecting improved scores for alcohol and placebo alcohol combined, as well as alcohol combined with energy drink showing improvement when contrasted with alcohol and energy drink placebo. Although with energy drink, improvements in reaction time approached significance.

These results clearly show the overall performanceimpairing effects of alcohol in this study, which then provide a useful basis from which to evaluate the subjective perception of impairment.

Subjective measures

The subjective response to both energy drink and alcohol followed a consistent pattern for four of the five subjective measures. Energy drink with alcohol placebo was associated with participants feeling more clearheaded and energetic, whilst less clumsy and drowsy. However, energy drink combined with alcohol resulted in participants feeling least clearheaded and energetic, together with being most clumsy, drowsy and mentally slow, precluding the significant effects for the energy drink treatment group compared to the placebo energy drink group. The results for the placebo energy drink group generally fell within these levels, with alcohol again increasing subjective impairment relative to alcohol placebo, although compared to the lower dose, the higher dose was associated with feeling more energetic. The general pattern of subjective impairment after alcohol for both the energy drink and placebo energy drink groups are plotted in Fig. 2, showing dose-response effects for the clearheaded and clumsy descriptors.

Statistical analysis supported the graphical presentations with alcohol producing significant increases in subjective impairment for feelings of being less clearheaded and more clumsy, drowsy and mentally slow (alcohol - clearheaded: $F_{1,18}=6.41, \mathrm{MSe}=761, P=0.02$; clumsy: $F_{1,18}=24.7, \mathrm{MSe}=$ 263, $P=0.001$; drowsy: $F_{1,18}=4.40$, MSe $=1193, P=0.05$; mentally slow: $\left.F_{1,18}=8.20, \mathrm{MSe}=148, P=0.01\right)$. Significant dose-related increases in perceived impairment between drink 1 to drink 2 were seen for clearheaded $\left(F_{1,18}=5.47\right.$, $\mathrm{MSe}=90.6, P=0.03)$, clumsy $\left(F_{1,18}=14.9, \mathrm{MSe}=80.5, P=\right.$ $0.001)$ and mentally slow $\left(F_{1,18}=4.61, \mathrm{MSe}=77.4, P=\right.$ 0.046 ). Whilst there were no overall significant differences between the energy drink and placebo energy drink groups, feelings of being energetic were associated with a drink $x$ treatment group interaction $\left(F_{1,18}=4.84, \mathrm{MSe}=151, P=\right.$ 0.04 ), as after the second drink, those in the energy drink group felt less energetic, whilst the placebo energy drink group felt more energetic. There was also an alcohol $\times$ drink interaction $\left(F_{1,18}=4.46, \mathrm{MSe}=90.8, P=0.049\right)$ with perceived drowsiness increasing after the second drink with alcohol compared to placebo alcohol.

Effect sizes of $\geq 0.2$ were again generally associated with statistically significant contrasts, although increased feelings of being drowsy and mentally slow that were experienced after the alcohol and energy drink combination contrasted with alcohol and energy drink placebo with effect sizes of $d>0.2$ 


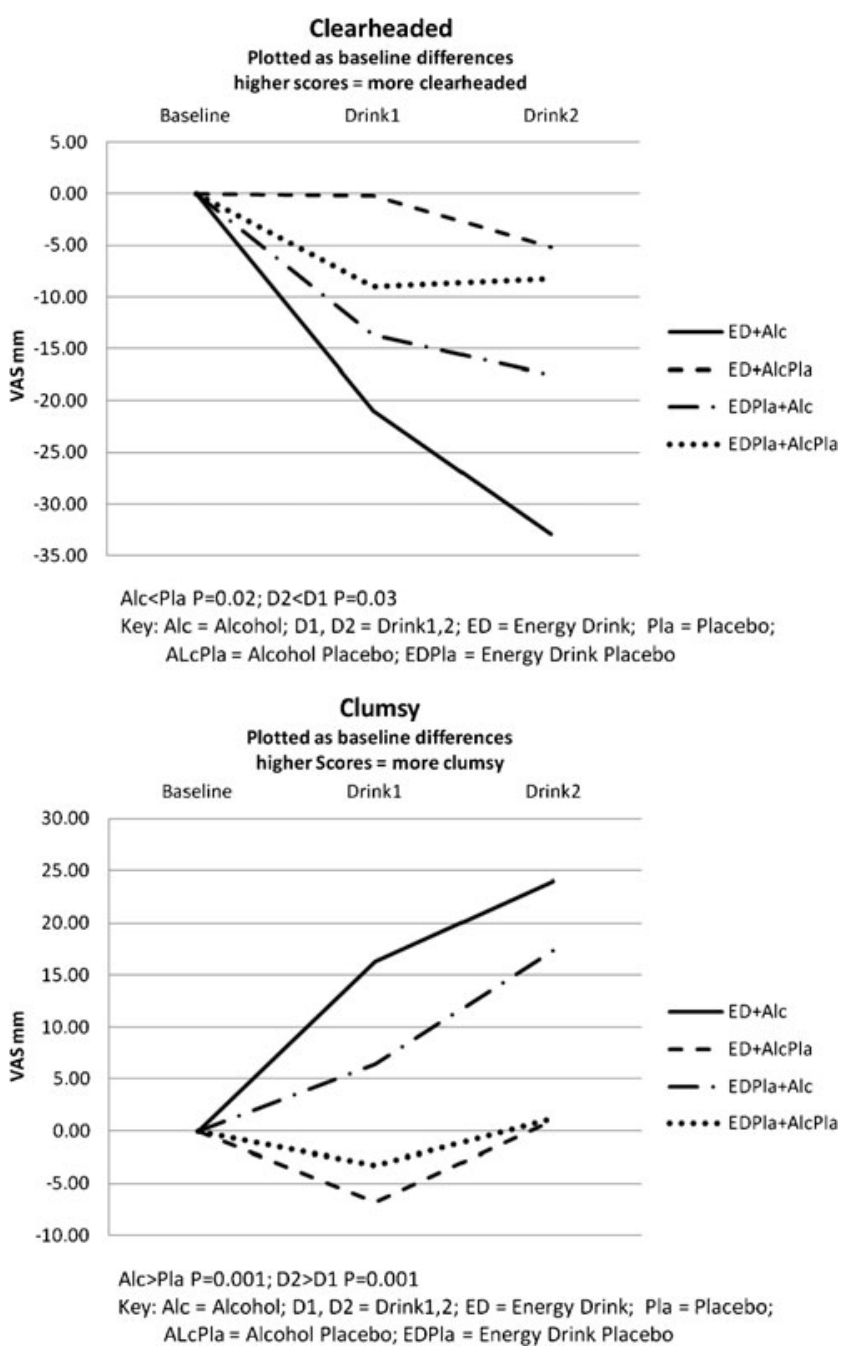

Fig. 2 Subjective awareness - clearheaded and clumsy, contrasting alcohol and energy drink alone, as well as in combination, and against their respective placebos

and associated $P<0.1$. Interestingly, the drink factor produced three significant contrasts between drink 1 and drink 2, although the associated effect sizes were $d<0.2$.

The subjective assessment revealed significant increases in impairment for four out of five descriptors with alcohol for the combined treatment groups and increases from drink 1 to drink 2 for three descriptors, but no overall significant differences between the energy drink and placebo energy drink groups. Significant interactions reflected a doserelated increase in drowsiness after alcohol and a reduction in feeling energetic after the second drink for the energy drink treatment group.

\section{Discussion}

The performance tests showed the expected impairment after alcohol. With laboratory tests, significant impairment is generally seen in $50 \%$ of studies at the $0.05 \% \mathrm{BAC}$ and $90 \%$ at the $0.08 \%$ BAC (Moskowitz and Fiorentino 2000), and therefore, similar to the concentration recorded here for the combined treatment groups $(0.046$ and $0.087 \% \mathrm{BrAC})$. The similarity in BrAC between the energy drink and placebo energy drink group allowed between-group comparisons for both alcohol doses and alcohol versus placebo comparisons.

Objective performance measures found statistically significant impairments with alcohol for both reaction time and memory, whilst CFF, which may be relatively insensitive to alcohol (Ogden and Moskowitz 2004), showed only trends for reduced flicker fusion thresholds with alcohol for both the energy drink and placebo energy drink groups. The results for Stroop performance were more complicated, with the greatest number of errors recorded at the higher dose for the placebo energy drink group, consistent with previous work reporting increased errors after alcohol (Tiplady et al. 2004, 2009). However, the combination of energy drink and alcohol significantly reduced completion time and errors, reflecting improved performance. Therefore, overall, alcohol was associated with impaired performance and greater impairment was seen at the higher dose for three out of four tasks.

Whilst studies assessing the effects of energy drink in combination with alcohol are very limited, it was noted in the 'Introduction' that the addition of caffeine has either antagonised the effects of alcohol, had no effect, or further increased impairment (Azcona et al. 1995; Liguori and Robinson 2001; Marczinski and Fillmore 2006; Nuotto et al. 1982; Oborne and Rogers 1983). From examining the means or graphical plots for the current study, energy drink and alcohol, when compared to placebo energy drink with alcohol, showed lower levels of impairment at the higher dose with CFF, total reaction time and RRT and a reduction in Stroop errors and completion time. However, no reduction was seen with memory.

These relative reductions in alcohol-induced impairment after coadministration of energy drink suggest at least some possible antagonism by caffeine or a combination of energy drink constituents. However, statistical evaluation only provided a trend $(P=0.06)$ for Stroop errors and a significant $(P=0.004)$ difference in completion time between the energy drink group and placebo energy drink group, although this may have been partially obscured by the combination of factors in the overall ANOVA. In comparison, the reduced simple main effects model demonstrated significant improvements for the alcohol and energy drink combination compared to alcohol and energy drink placebo for both Stroop errors $(P=0.028)$ and completion time $(P=0.004)$.

The notable improvement in Stroop completion time seen here, also reflecting a reduction in errors, when alcohol was combined with energy drink may reflect an anomaly of the 
Stroop assessment. Participants were asked to name the ink colour and ignore the printed word. There are different possible assessments that can be undertaken (Stroop 1935); for example, participants could have been asked to name the printed word and ignore the ink colour. By asking participants to name the ink colour and ignore the printed word, a reduction in focus or visual acuity as occurs with alcohol (Moskowitz et al. 1993) would aid perception and dominance of the ink colour as the printed letters became potentially more ambiguous. This would reduce the interference effect and possibly improve performance. However, greatest errors were seen after the higher dose of alcohol in the placebo energy drink group, though speed was not increased, supporting earlier findings of increased errors after alcohol (Starmer 1989; Tiplady et al. 2009). In contrast, Liguori and Robinson (2001) failed to find significant effects with either CFF or Stroop in their investigation of caffeine and alcohol.

The results for word memory were stronger and more consistent with both immediate and delayed recall producing highly significant results $(P=0.001)$ for alcohol impairment and increase with the higher dose. There was no evidence of energy drink antagonism of alcohol impairment. The results with alcohol are generally in keeping with the literature as memory impairment is a robust and common feature of alcohol intoxication (Maylor and Rabbitt 1993) and provide further support for the results of the current study.

Therefore, three out of four tests showed either trends (CFF) or significant impairment (reaction time, word memory) after alcohol, but no significant differences between the energy drink or placebo energy drink groups. One test (Stroop) showed a significant improvement in both completion time and errors after the alcohol and energy drink combination.

The five scales selected from the Bond and Lader (1974) VAS scales were comprised of bipolar opposites (e.g. alertdrowsy), with the midpoint representing a neutral position. The scales are suitable for assessing both stimulant (e.g. more alerting) as well as sedative (e.g. increased drowsiness) effects and are, therefore, suitable for assessing both the individual and combined effects of caffeine-based energy drinks (stimulant) and alcohol (sedative), although noting that rising blood alcohol levels can be associated with feelings of increased activation (Rueger et al. 2009).

The results showed significant and consistent increases in subjective impairment after alcohol for four out of five scales, although feelings of being energetic showed a dose-related reduction with alcohol in the energy drink group but contrasted with an increase after the second drink for the placebo energy drink group, precluding an overall effect of alcohol for the two groups combined. Dose-related increases in subjective impairment were reflected in significant increases for the drinks factor with three of the five descriptors (less clearheaded, more clumsy and mentally slow), whilst a significant interaction between alcohol and drink reflected the increase in drowsiness after the second alcohol drink for both treatment groups combined.

There was no evidence of antagonism of alcohol-induced subjective impairment by energy drink as there were no overall significant differences between the energy drink and placebo energy drink groups, nor specifically between the alcohol and energy drink combination compared to alcohol and energy drink placebo with simple main effects analysis. Although, as mentioned, feelings of energy were decreased for the energy drink group after the second drink compared to the placebo energy drink, reflecting a divergence in the perception of alcohol-induced sedation between groups. Overall, in comparison to the other treatment conditions, energy drink without alcohol consistently resulted in the least levels of sedation or greatest subjective stimulation for all but the mentally slow descriptor. In contrast, energy drink combined with alcohol produced the highest levels of subjective impairment and sedation including effect sizes of $d \geq 0.2$ for drowsy and mentally slow, although this may partially reflect differences between the two participant groups.

What is clear is that the subjective perception of impairment was reliably and consistently seen after alcohol and that several descriptors were sensitive to the increase in dose. None of the descriptors showed overall significant differences between the energy drink combined with alcohol and alcohol alone. There was no evidence of a possible masking effect with energy drink, reducing the perception of intoxication; indeed, the mean trends were for the energy drink and alcohol combination to show greater levels of subjective impairment compared to alcohol alone, although differences were not statistically significant.

These findings receive some support from the recent study by Marczinski et al. (2011) who compared the effects of alcohol alone and in combination with energy drink assessing breath alcohol, performance and subjective measures. They also found no significant differences between breath alcohol levels, performance and for subjective effects including intoxication and ability to drive with ANOVA. However, paired comparisons showed reduced subjective mental fatigue and increased stimulation for the alcohol and energy drink combination compared to alcohol alone. These 2 findings, out of over 20 individual ratings taken, are in contrast to the present study where mean values for energetic and mentally slow were not reduced with the alcohol and energy drink combination.

These results clearly contrast with the only other currently published study directly comparing the effects of alcohol and energy drink alone and in combination on both psychomotor performance and subjective intoxication. The study of 
Ferreira et al. (2006) interpreted the findings as demonstrating that energy drink reduced the perception of alcohol intoxication although they had more descriptors registering impairment with the energy drink and alcohol combination, as well as alcohol alone, than showed reduced impairment after coadministration of energy drink with alcohol. Therefore, their overall results were more in line with the findings of the present study. Other similarities between these two studies include the two alcohol levels. Further, the findings of significant objective performance impairment in both studies provided a suitable basis from which to assess subjective awareness of impairment. One difference between the studies was that, in the Ferreira study, one participant group received the lower alcohol dose and the other the higher dose, whilst in the present study, participant groups were split by energy drink and placebo energy drink.

The sample size of 10 participants per group is a limitation with the present study through limiting power which may then impact on the number of statistically significant contrasts observed and requiring caution in interpreting these results. However, the results showed that the study was sufficiently powered to produce significant contrasts for all three factors analysed, i.e. alcohol compared to placebo alcohol, energy drink compared to placebo energy drink, and drink 1 compared to drink 2, as well as significant interactions between them. The inclusion of effect size values (Cohen's $d$ ) found that, generally, effect sizes of $d \geq 0.2$, and therefore classified as at least 'small' (Cohen 1992), were associated with statistically significant effects, although improvements in reaction time after energy drink were associated with effect sizes of $d \geq 0.2$ but failed to achieve significance $(P=0.06)$. Similarly, increased feelings of drowsiness and being mentally slow for the alcohol and energy drink combination compared to alcohol also achieved the 'small' effect size threshold but failed to achieve significance $(P<0.1)$. These might reflect sample size as well as between-group differences.

Future studies might benefit from using a larger sample to increase power and a fully repeated-measures design to enable more sensitive comparisons between all four treatment combinations of alcohol, energy drink and their respective placebos, although repeated testing can itself impact on results.

Indirect support for our findings comes from studies investigating the combined effect of caffeine and alcohol compared to alcohol alone, including higher alcohol and caffeine concentrations than investigated here, but also failing to find any difference in either BAC or perception of alcohol intoxication. These laboratory investigations have shown that caffeine and alcohol in combination do not alter the 'high' or perceived or actual BAC with caffeine levels up to around $400 \mathrm{mg}$ and alcohol 0.012\% BAC (Howland et al. 2010; Liguori and Robinson 2001; Rush et al. 1993).
In conclusion, the present study found that alcohol at doses of 0.046 to $0.087 \%$ BrAC impaired psychomotor and cognitive performance. The combination of energy drink with alcohol failed to show consistent differences from alcohol alone on several performance measures, although Stroop performance was improved. The combination of energy drink with alcohol did not change subjective perceptions of impairment when contrasted with alcohol alone. There was no evidence of a reduced perception of impairment or masking effect for energy drink combined with alcohol compared to alcohol alone.

Conflict of interest Over the last 3 years, Chris Alford has received funding from the UK Ministry of Defence, Red Bull GmbH and Sanofi-Aventis and was scientific adviser to Red Bull GmbH and Sanofi-Aventis, Japan. Joris Verster has received research funding from Takeda Pharmaceuticals and Red Bull GmbH and was scientific advisor for Takeda, Sanofi-Aventis, Transcept, Sepracor, Red Bull GmbH, Deenox, Trimbos Institute and CBD.

Funding This study was undertaken at the University of the West of England without support from a sponsor.

\section{References}

Adan A, Serra-Grabulosa JM (2011) Effect of caffeine and glucose, alone and combined, on cognitive performance. Human Psychopharmacol 25:310-317

Alford C (2009) Sleepiness, countermeasures and the risk of motor vehicle accidents. In: Verster JC, Pandi-Perumal SR, Rameakers JG, De Gier JJ (eds) Drugs, driving and traffic safety. Birkhauser, Boston, pp 207-232

Alford C, Cox H, Wescott R (2001) The effects of red bull energy drink on human performance and mood. Amino Acids 21:139-150

APA (1994) Diagnostic and statistical manual of mental disorders (DSM-IV), 4th edn. American Psychiatric Association, Arlington

Arria AM, O'Brien MC (2011) The "high" risk of energy drinks. JAMA 305:600-601

Arria AM, Caldeira KM, Kasperski SJ, O’Grady KE, Vincent KB, Griffiths RR, Wish ED (2010) Increased alcohol consumption, nonmedical prescription drug use, and illicit drug use are associated with energy drink consumption among college students. J Addict Med 4:74-80

Astorino TA, Roberson DW (2010) Efficacy of acute caffeine ingestion for short-term high-intensity exercise performance: a systematic review. J Strength Cond Res 24:257-265

Attila S, Cakir B (2010) Energy-drink consumption in college students and associated factors. Nutrition 27:316-322

Azcona O, Barbanoj MJ, Torrent J, Jane F (1995) Evaluation of the central effects of alcohol and caffeine interaction. Br J Clin Pharmacol 40:393-400

Berger LK, Fendrich M, Chen HY, Arria AM, Cisler RA (2010) Sociodemographic correlates of energy drink consumption with and without alcohol: results of a community survey. Addict Behav $36: 516-519$ 
Betts T, Markman D, Debenham S, Mortiboy D, McKevitt T (1984) Effects of two antihistamine drugs on actual driving performance. Br Med J (Clin Res Ed) 288:281-282

Bond A, Lader M (1974) Use of analog scales in rating subjective feelings. Br J Med Psychol 47:211-218

Borkenstein R, Crowther RF, Shumate RP, Zeil WW, Zylinan R (1964) The role of the drinking driver in traffic accidents. Department of Police Administration, Indiana University, Bloomington

Braun S (1996) Buzz. Oxford University Press, New York

Cameron E, Sinclair W, Tiplady B (2001) Validity and sensitivity of a pen computer battery of performance tests. J Psychopharmacol 15:105-110

Christopher G, Sutherland D, Smith A (2005) Effects of caffeine in non-withdrawn volunteers. Hum Psychopharmacol 20:47-53

Cohen J (1992) A power primer. Psychol Bull 112:155-159

Cohen J, Dearnaley EJ, Hansel CE (1958) The risk taken in driving under the influence of alcohol. Br Med J 1:1438-1442

Davis JM, Zhao Z, Stock HS, Mehl KA, Buggy J, Hand GA (2003) Central nervous system effects of caffeine and adenosine on fatigue. Am J Physiol Regul Integr Comp Physiol 284:R399R404

Fagan D, Swift CG, Tiplady B (1998) Effects of caffeine on vigilance and other performance tests in normal subjects. J Psychopharmacol 2:19-25

Farquhar K, Lambert K, Drummond GB, Tiplady B, Wright P (2002) Effect of ethanol on psychomotor performance and on risk taking behaviour. J Psychopharmacol 16:379-384

Ferre $\mathrm{S}$ (2008) An update on the mechanisms of the psychostimulant effects of caffeine. J Neurochem 105:1067-1079

Ferreira SE, de Mello MT, Rossi MV, Souza-Formigoni ML (2004) Does an energy drink modify the effects of alcohol in a maximal effort test? Alcohol Clin Exp Res 28:1408-1412

Ferreira SE, de Mello MT, Pompeia S, de Souza-Formigoni ML (2006) Effects of energy drink ingestion on alcohol intoxication. Alcohol Clin Exp Res 30:598-605

Fillmore MT, Vogel-Sprott M (1995) Behavioral effects of combining alcohol and caffeine: contribution of drug-related expectancies. Exp Clin Psychopharmacol 3:33-38

Fillmore MT, Roach EL, Rice JT (2002) Does caffeine counteract alcohol-induced impairment? The ironic effects of expectancy. J Stud Alcohol 63:745-754

Flanagan NG, Strike PW, Rigby CJ, Lochridge GK (1983) The effects of low doses of alcohol on driving performance. Med Sci Law 23:203-208

Frewer LJ, Lader M (1991) The effects of caffeine on 2 computerized tests of attention and vigilance. Human Psychopharmacol Clin Exp 6:119-128

Fromme K, D'Amico EJ, Katz EC (1999) Intoxicated sexual risk taking: an expectancy or cognitive impairment explanation? J Stud Alcohol 60:54-63

Gershon P, Shinar D, Ronen A (2009) Evaluation of experience-based fatigue countermeasures. Accid Anal Prev 41:969-975

Glade MJ (2010) Caffeine - not just a stimulant. Nutrition 26:932-938

Halpern-Fischer BL, Millstein SG, Ellen JM (1996) Relationship of alcohol use and risky sexual behavior: a review and analysis of findings. J Adolesc Health 19:331-336

Hasenfratz M, Bunge A, Dal Pra G, Battig K (1993) Antagonistic effects of caffeine and alcohol on mental performance parameters. Pharmacol Biochem Behav 46:463-465

Heckman MA, Sherry K, de Mejia EG (2010) Energy drinks: an assessment of their market size, consumer demographics, ingredient profile, functionality, and regulations in the United States. Compr Rev Food Sci Food Saf 9:303-317

Hindmarch I, Kerr JS, Sherwood N (1991) The effects of alcohol and other drugs on psychomotor performance and cognitive function. Alcohol Alcohol 26:71-79
Hindmarch I, Alford C, Barwell F, Kerr J (1992) Measuring the side effects of psychotropics: the behavioural toxicity of antidepressants. J Psychopharmacol 6:198-203

Hoffman JR (2010) Caffeine and energy drinks. Strength Cond J 20:15-20

Hollingworth HL (1912) The influence of caffeine on the speed and quality of performance in typewriting. Psychol Rev 19:66-73

Holloway FA (1995) Low-dose alcohol effects on human behaviour and performance. Alcohol Drugs Driving 11:39-56

Horne JA, Reyner LA (2001) Beneficial effects of an "energy drink" given to sleepy drivers. Amino Acids 20:83-89

Howland J, Rohsenow DJ, Arnedt JT, Bliss CA, Hunt SK, Calise TV, Heeren T, Winter M, Littlefield C, Gottlieb DJ (2010) The acute effects of caffeinated versus non-caffeinated alcoholic beverage on driving performance and attention/reaction time. Addiction 106:335-341

Huang ZL, Urade Y, Hayaishi O (2007) Prostaglandins and adenosine in the regulation of sleep and wakefulness. Curr Opin Pharmacol 7:33-38

IFIC (1998) IFIC review: caffeine and health: clarifying the controversies. IFIC Foundation, Washington, DC

Ivy JL, Kammer L, Ding Z, Wang B, Bernard JR, Liao YH, Hwang J (2009) Improved cycling time-trial performance after ingestion of a caffeine energy drink. Int $\mathrm{J}$ Sport Nutr Exerc Metab 19:61-78

Jääskeläinen IP, Pekkonen E, Alho K, Sinclair JD, Sillanaukee P, Näätänen R (1995) Dose-related effect of alcohol on mismatch negativity and reaction time performance. Alcohol 12:491-495

James JE (1997) Understanding caffeine: a behavioral analysis. Sage, Thousand Oaks

James JE, Gregg ME (2004) Effects of dietary caffeine on mood when rested and sleep restricted. Hum Psychopharmacol 19:333-341

James JE, Rogers PJ (2005) Effects of caffeine on performance and mood: withdrawal reversal is the most plausible explanation. Psychopharmacology (Berl) 182:1-8

Ker K, Edwards PJ, Felix LM, Blackhall K, Roberts I (2010) Caffeine for the prevention of injuries and errors in shift workers. Cochrane Database Syst Rev (5):CD008508

Klepacki B (2010) Energy drinks: a review article. Strength Cond J $32: 37-41$

Koelega HS (1995) Alcohol and vigilance performance: a review. Psychopharmacology (Berl) 118:233-249

Koelega HS (1998) Effects of caffeine, nicotine and alcohol on vigilance performance. In: Snel J, Lorist M (eds) Nicotine, caffeine and social drinking. OPA, Amsterdam, pp 363-373

Landolt HP (2008) Sleep homeostasis: a role for adenosine in humans? Purinergic Signal 4:S115-S116

Liguori A, Robinson JH (2001) Caffeine antagonism of alcoholinduced driving impairment. Drug Alcohol Depend 63:123-129

Lovinger DM (2008) Communication networks in the brain: neurons, receptors, neurotransmitters, and alcohol. Alcohol Res Health $31: 196-214$

Mackay M, Tiplady B, Scholey AB (2002) Interactions between alcohol and caffeine in relation to psychomotor speed and accuracy. Human Psychopharmacol Clin Exp 17:151-156

Malinauskas BM, Aeby VG, Overton RF, Carpenter-Aeby T, BarberHeidal K (2007) A survey of energy drink consumption patterns among college students. Nutr J 6:35

Marczinski CA, Fillmore MT (2006) Clubgoers and their trendy cocktails: implications of mixing caffeine into alcohol on information processing and subjective reports of intoxication. Exp Clin Psychopharmacol 14:450-458

Marczinski CA, Fillmore MT, Henges AL, Ramsey MA, Young CR (2011) Effects of energy drinks mixed with alcohol on information processing, motor coordination and subjective reports of intoxication. Exp Clin Psychopharmacol. doi:10.1037/a0026136 
Maylor EA, Rabbitt PM (1993) Alcohol, reaction time and memory: a meta-analysis. Br J Psychol 84(Pt 3):301-317

McKim WA (1997) Drugs and behaviour, 3rd edn. Prentice Hall Inc., Upper Saddle River

Mets MA, Ketzer S, Blom C, van Gerven MH, van Willigenburg GM, Olivier B, Verster JC (2010) Positive effects of Red Bull ${ }^{\circledR}$ Energy Drink on driving performance during prolonged driving. Psychopharmacology (Berl) 214:737-745

Millar SA, Duncan L, Tiplady B (1995) Ethanol-induced CNS depression and divided attention. Hum Psychopharmacol 10:327-331

Miller E (1992) Effect of moderate alcohol intake on cognitive functioning: does a little bit of what you fancy do you good? J Ment Health 1:19-24

Moskowitz H, Fiorentino D (2000) A review of the scientific literature regarding the effects of alcohol on driving-related behavior at blood alcohol concentrations of $80 \mathrm{mg} / \mathrm{dl}$ and lower (report HS809-028). US Department of Transportation, National Highway Traffic Safety Administration, Washington, DC

Moskowitz H, Burns MM, Williams AF (1985) Skills performance at low blood alcohol levels. J Stud Alcohol 46:482-485

Moskowitz H, Wilkinson C, Burg A (1993) The effect of alcohol on driving related visual performance. In: Utzelmann $\mathrm{H}$, Berghaus $\mathrm{G}$, Kroj G (eds) Alcohol, drugs and traffic safety-T92. Proceedings of the 12th International Conference on Alcohol, Drugs and Traffic Safety. Verlog TUV Rheinland, Cologne

Mumford GK, Evans SM, Kaminski BJ, Preston KL, Sannerud CA, Silverman K, Griffiths RR (1994) Discriminative stimulus and subjective effects of theobromine and caffeine in humans. Psychopharmacology (Berl) 115:1-8

Nehlig A (2010) Is caffeine a cognitive enhancer? J Alzheimers Dis 20 (Suppl 1):S85-S94

Nehlig A, Daval JL, Debry G (1992) Caffeine and the central nervous system: mechanisms of action, biochemical, metabolic and psychostimulant effects. Brain Res Brain Res Rev 17:139-170

Nuotto E, Mattila MJ, Seppala T, Konno K (1982) Coffee and caffeine and alcohol effects on psychomotor function. Clin Pharmacol Ther 31:68-76

Oborne DJ, Rogers Y (1983) Interactions of alcohol and caffeine on human reaction time. Aviat Space Environ Med 54:528-534

O'Brien MC, McCoy TP, Rhodes SD, Wagoner A, Wolfson M (2008) Caffeinated cocktails: energy drink consumption, high-risk drinking, and alcohol-related consequences among college students. Acad Emerg Med 15:453-460

Ogden EJ, Moskowitz H (2004) Effects of alcohol and other drugs on driver performance. Traffic Inj Prev 5:185-198

Oteri A, Salvo F, Caputi AP, Calapai G (2007) Intake of energy drinks in association with alcoholic beverages in a cohort of students of the School of Medicine of the University of Messina. Alcohol Clin Exp Res 31:1677-1680

Paivio A, Yuille JC, Madigan SA (1968) Concreteness, imagery, and meaningfulness values for 925 nouns. J Exp Psychol 76 (Suppl): $1-25$

Reissig CJ, Strain EC, Griffiths RR (2009) Caffeinated energy drinks - a growing problem. Drug Alcohol Depend 99:1-10

Reyner LA, Horne JA (2002) Efficacy of a 'functional energy drink' in counteracting driver sleepiness. Physiol Behav 75:331-335

Riesselmann B, Rosenbaum F, Schneider V (1996) Alcohol and energy drink - can combined consumption of both beverages modify automobile driving fitness? Blutalkohol 33:201-208

Ritchie JM (1980) Central nervous stimulants, the xanthines. In: Gillman A, Goodman L (eds) The pharmacological basis of therapeutics. MacMillan Publishing Company, New York

Rivers PC (1994) Alcohol and human behaviour: theory, research and practice. Prentice Hall Inc., Upper Saddle River

Robelin M, Rogers PJ (1998) Mood and psychomotor performance effects of the first, but not of subsequent, cup-of-coffee equivalent doses of caffeine consumed after overnight caffeine abstinence. Behav Pharmacol 9:611-618

Rogers PJ, Heatherley SV, Hayward RC, Seers HE, Hill J, Kane M (2005) Effects of caffeine and caffeine withdrawal on mood and cognitive performance degraded by sleep restriction. Psychopharmacology (Berl) 179:742-752

Rossheim ME, Thombs DL (2011) Artificial sweeteners, caffeine, and alcohol intoxication in bar patrons. Alcohol Clin Exp Res 35:1891-1896

Rueger SY, McNamara PJ, King AC (2009) Expanding the utility of the Biphasic Alcohol Effects Scale (BAES) and initial psychometric support for the Brief-BAES (B-BAES). Alcohol Clin Exp Res 33:916-924

Rush CR, Higgins ST, Hughes JR, Bickel WK, Wiegner MS (1993) Acute behavioral and cardiac effects of alcohol and caffeine, alone and in combination, in humans. Behav Pharmacol 4:562-572

Schilling W (1921) The effect of caffein and acetanilid on simple reaction time. Psychol Rev 28:72-79

Scholey AB, Kennedy DO (2004) Cognitive and physiological effects of an "energy drink": an evaluation of the whole drink and of glucose, caffeine and herbal flavouring fractions. Psychopharmacology 176:320-330

Seidl R, Peyrl A, Nicham R, Hauser E (2000) A taurine and caffeinecontaining drink stimulates cognitive performance and wellbeing. Amino Acids 19:635-642

Siegel S (2011) The four-loko effect. Perspect Psychol Sci 6:357-362

Smit HJ, Rogers PJ (2000) Effects of low doses of caffeine on cognitive performance, mood and thirst in low and higher caffeine consumers. Psychopharmacology 152:167-173

Smit HJ, Grady ML, Finnegan YE, Hughes SA, Cotton JR, Rogers PJ (2006) Role of familiarity on effects of caffeine- and glucosecontaining soft drinks. Physiol Behav 87:287-297

Smith A (2002) Effects of caffeine on human behavior. Food Chem Toxicol 40:1243-1255

Smith A, Sutherland D, Christopher G (2005) Effects of repeated doses of caffeine on mood and performance of alert and fatigued volunteers. J Psychopharmacol 19:620-626

Sokmen B, Armstrong L, Kraemer WJ, Casa DJ, Dias JC, Judelson DA, Maresh CM (2008) Caffeine use in sports: considerations for the athlete. J Strength Cond Res 22:978-986

SPSS (2010) SPSS version 17. IBM, SPSS Inc., Chicago

Starmer G (1989) Effects of low to moderate doses of ethanol on human driving performance. In: Crow K, Batt R (eds) Human metabolism of alcohol (volume 1): pharmacokinetics, medicolegal aspects and general interest. CRC, Boca Raton

Stroop JR (1935) Studies of interference in serial verbal reactions. J Exp Psychol 18:643-662

Sturgess JE, Ting AKRA, Podbielski D, Sellings LH, Chen JF, van der Kooy D (2010) Adenosine A1 and A2A receptors are not upstream of caffeine's dopamine D2 receptor-dependent aversive effects and dopamine-independent rewarding effects. Eur J Neurosci 32:143-154

Swift CG, Tiplady B (1988) The effects of age on the response to caffeine. Psychopharmacology (Berl) 94:29-31

Tagawa M, Kano M, Okamura N, Itoh M, Sakurai E, Watanabe T, Yanai K (2000) Relationship between effects of alcohol on psychomotor performances and blood alcohol concentrations. Jpn J Pharmacol 83:253-260

Thombs DL, O'Mara RJ, Tsukamoto M, Rossheim ME, Weiler RM, Merves ML, Goldberger BA (2010a) Event-level analyses of energy drink consumption and alcohol intoxication in bar patrons. Addict Behav 35:325-330

Thombs DL, Rossheim M, Barnett TE, Weiler RM, Moorhouse MD, Coleman BN (2010b) Is there a misplaced focus on AmED? Associations between caffeine mixers and bar patron intoxication. Drug Alcohol Depend 116:31-36 
Tiplady B, Franklin N, Scholey A (2004) Effect of ethanol on judgments of performance. Br J Psychol 95:105-118

Tiplady B, Oshinowo B, Thomson J, Drummond GB (2009) Alcohol and cognitive function: assessment in everyday life and laboratory settings using mobile phones. Alcohol Clin Exp Res 33:2094-2102

Van der Stelt O, Snel J (1998) Caffeine and human performance. In: Snel J, Lorist $M$ (eds) Nicotine, caffeine and social drinking. OPA, Amsterdam

Verster JC, Alford C (2011) Unjustified concerns about energy drinks. Current Drug Abuse Rev 4:1-3

Verster JC, Wester AE, Goorden M, van Wieringen JP, Olivier B, Volkerts ER (2009) Novice drivers' performance after different alcohol dosages and placebo in the divided-attention steering simulator (DASS). Psychopharmacology (Berl) 204:127-133
Wallgren H, Barry H (1970) Actions of alcohol. Elsevier, New York Walsh AL, Gonzalez AM, Ratamess NA, Kang J, Hoffman JR (2010) Improved time to exhaustion following ingestion of the energy drink Amino Impact. J Int Soc Sports Nutr 7:14

Warburton DM (1995) Effects of caffeine on cognition and mood without caffeine abstinence. Psychopharmacology (Berl) 119:66-70

Warburton DM, Bersellini E, Sweeney E (2001) An evaluation of a caffeinated taurine drink on mood, memory and information processing in healthy volunteers without caffeine abstinence. Psychopharmacology (Berl) 158:322-328

Watson J, Deary I, Kerr D (2002) Central and peripheral effects of sustained caffeine use: tolerance is incomplete. Br J Clin Pharmacol 54:400-406 